\title{
Contribution de la physique au développement pharmaceutique de nouveaux médicaments
}

Michel Bauer (michel.bauer@sanofi-aventis.com)

Sanofi-aventis, Centre de recherche et développement, 34184 Montpellier Cedex 04

La recherche

et le développement

pharmaceutiques

modernes font largement

appel à la physique

et à la modélisation

mathématique,

que ce soit pour la

conception de nouvelles

entités actives

ou le développement

de formes libérant

leurs principes actifs

de façon programmée

ou ciblée. Cet article

illustre ce propos à partir

de trois exemples

d'axes de recherche

pharmaceutique : la

conception de molécules

antithrombotiques,

la mise en place d'un

contrôle de qualité de

comprimés à libération

prolongée, la conception

de médicaments mieux

absorbés par l'organisme.

Les termes suivis d'un astérisque (* sont définis dans le glossaire, p.9.
Il y a un temps, encore pas si lointain, où la création, le développement et l'industrialisation du médicament relevaient plus de l'art et de l'empirisme que d'une réflexion rigoureuse se nourrissant de l'apport des sciences fondamentales comme la physique ou les mathématiques. Aujourd'hui, la situation a notablement changé. Une partie non négligeable des nouveaux médicaments mis sur le marché consiste en des entités moléculaires conçues grâce à la chimie moléculaire quantique. Par ailleurs, l'apport de méthodes physiques de plus en plus performantes (RX, RMN, spectrométrie de masse, spectroscopies IR et Raman, microscopie sous toutes ses formes...) a permis de connaître de mieux en mieux les caractéristiques des principes actifs et des médicaments qui en sont issus. En d'autres termes, le développement pharmaceutique moderne est devenu un champ d'application tout à fait considérable de la physique et de la chimie des solides pulvérulents, ainsi que de la modélisation mathématique.

Il faut bien saisir, ce qui n'est en général pas connu du " grand public ", qu'un médicament ne consiste pas seulement en une ou plusieurs entités pharmacologiquement et cliniquement actives dans une ou plusieurs pathologies. Ce qu'un patient absorbe, dans la majorité des cas encore aujourd'hui, c'est une préparation solide absorbable par voie orale. Elle résulte d'une association parfaitement étudiée d'un principe actif et d'un certain nombre de produits inactifs solides, les " excipients ", destinés à assurer la fabrication à une échelle industrielle, la stabilité physico-chimique du principe actif, ainsi que sa biodisponibilité $e^{*-*}$.

Nous allons tenter d'illustrer les propos précédents à partir de trois exemples. Le premier exemple souligne l'intérêt de l'utilisation de la diffraction des rayons $\mathrm{X}$ sur monocristaux de protéines dans la conception de molécules présentant une activité antithrombotique après administration par voie orale. Le deuxième décrit comment l'utilisation de la modélisation permet la mise en place d'un contrôle de qualité d'un lot de comprimés libérant dans l'organisme leur principe actif de façon prolongée. Enfin, le troisième exemple montre comment une technique physique comme la spectroscopie diélectrique peut apporter une aide précieuse dans la conception de médicaments mieux absorbés par l'organisme.

\section{Apport de la diffraction} des RX sur monocristaux de protéines dans la conception de molécules antithrombotiques

$\mathrm{La}$ thrombose consiste en la formation accidentelle d'un caillot sanguin dans un vaisseau, pouvant entraîner un risque de décès. Ce caillot est l'aboutissement d'un processus compliqué, comportant un nombre important de réactions biochimiques catalysées par des enzymes protéiques appelées facteurs de coagulation. L'ensemble de ces réactions constitue la « cascade de la coagulation " (fig. 1). Dans le sang circule le fibrinogène, qui est une grosse protéine, de masse moléculaire supérieure à 100 000. Celle-ci va être transformée en une protéine filamenteuse, la fibrine, dont les filaments vont ultérieurement subir des réactions croisées et former un réticulum qui est justement le caillot sanguin. La transformation du fibrinogène en fibrine est catalysée par une enzyme, la thrombine, qui elle-même n'est pas présente en tant que telle dans le sang, mais provient d'une protéine, la prothrombine, sous l'action d'une réaction

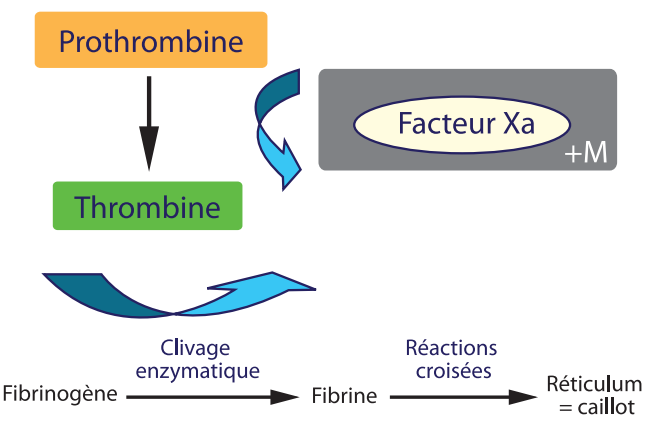

Figure 1 : Schéma d'une partie de la cascade de coagulation montrant le caractère direct du mécanisme d'action de la molécule M sur le facteur Xa qui catalyse la transformation de la prothrombine en thrombine. 


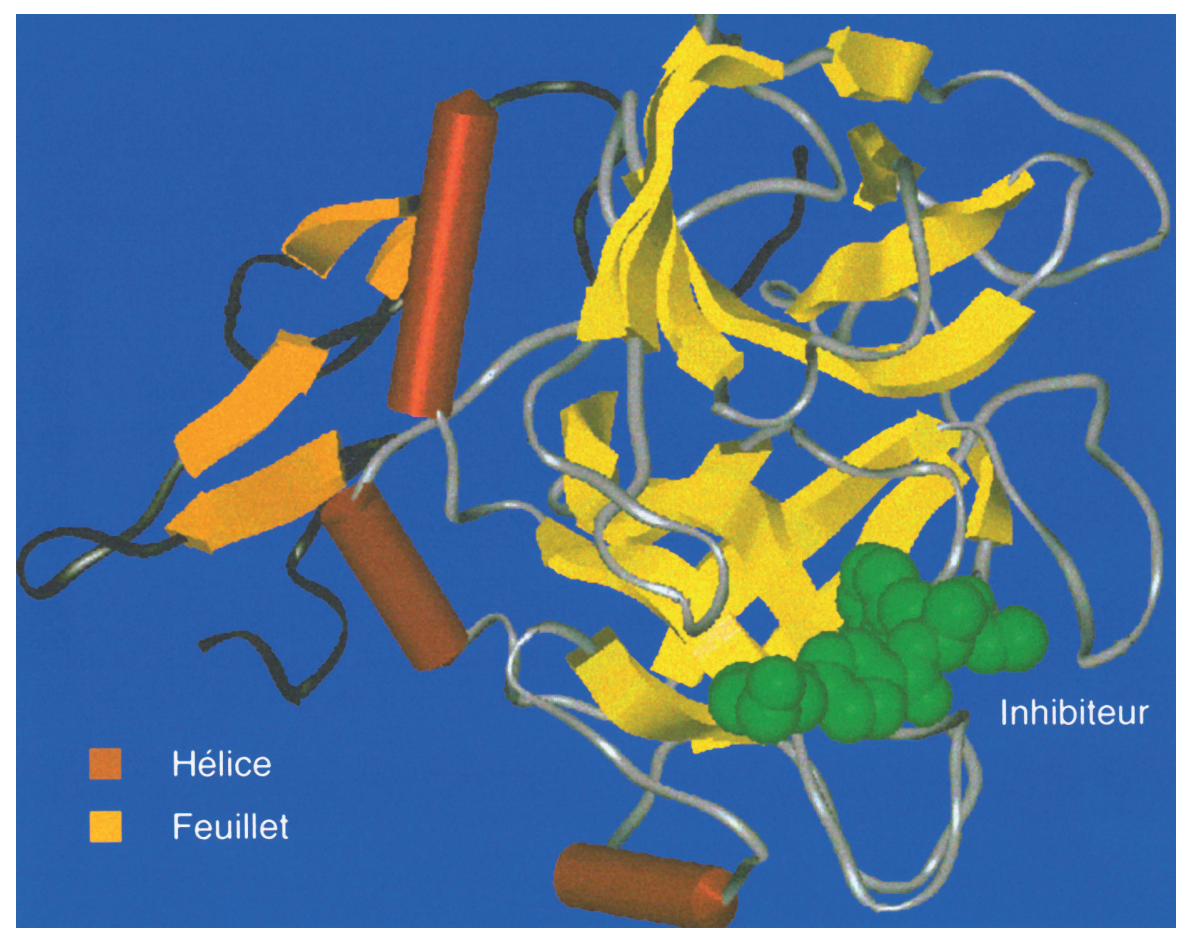

Figure 2 : Structure symbolique du facteur Xa avec, en vert, la zone d'interaction avec les inhibiteurs. Les tubes en orange symbolisent les régions sous forme d'hélices et les bandes jaunes, celles sous forme de feuillets plissés.

enzymatique catalysée par une autre protéine, nommée facteur Xa (fXa). Une des pistes de recherche consiste à concevoir des molécules organiques venant bloquer spécifiquement le fXa et donc le processus de coagulation. Compte tenu du positionnement du facteur Xa dans la cascade de coagulation, ce type d'inhibiteur entraîne en principe moins de risques de saignements, contrairement à d'autres inhibiteurs agissant à d'autres points clefs du processus. Comme la majorité des protéines impliquées dans la régulation des phénomènes biologiques, le $\mathrm{fX}$ a possède un domaine localisé où des molécules peuvent venir se fixer et inhiber en conséquence son activité biologique.

La seconde moitié du $20^{\mathrm{e}}$ siècle a vu une croissance exponentielle dans la détermination de structure des protéines à l'aide de la diffraction des RX sur monocristaux. La connaissance empirique de molécules inhibitrices a permis de déterminer par cette même approche les zones réceptrices où s'opère leur interaction avec la protéine. La figure 2 représente sous forme symbolique la structure du $\mathrm{fXa}$, déterminée par diffraction X sur monocristal, avec, en vert, la zone réceptrice des molécules inhibitrices directes de ce facteur de la coagulation.

Des molécules inhibitrices du $\mathrm{fXa}$ ont ainsi été conçues en s'aidant de la géométrie de la zone de réception et de sa composition en aminoacides. La première molécule envisagée $\left(M_{1}\right)$ comportait un groupement benzamidine hydrophile assurant une grande affinité pour le fXa. Malheureusement, cette molécule ne peut être administrée par voie orale, car elle n'est pas suffisamment lipophile pour traverser la barrière bilipidique séparant l'intestin des vaisseaux sanguins. Il a donc fallu développer de nouvelles molécules dotées d'une biodisponibilité suffisante $[1,2]$. Un premier tri a été effectué à l'aide d'un test biologique in vitro, qui consiste à mesurer la constante d'affinitév' $\mathrm{K}_{\mathrm{D}}$ du complexe protéine-ligand formé. Nous présentons l'exemple d'une molécule $\left(\mathrm{M}_{2}\right)$ où le groupement benzamidine a été remplacé par un groupement azaindole, moins polaire, et pour laquelle le couple fXa-M $\mathrm{M}_{2}$ présente un $\mathrm{K}_{\mathrm{D}}=18 \mathrm{nmol}^{-1}{ }^{-1}$, ce qui est une valeur assez faible pour en faire un bon candidat pour un développement clinique ultérieur. La figure 3 montre la structure de ce complexe, déterminée par diffraction $\mathrm{X}$ sur monocristal, et comment la molécule $\left(\mathrm{M}_{2}\right)$ s'adapte bien dans la zone réceptrice. La stabilisation énergétique du complexe se trouve assurée d'une part, par les forces de Van der Waals entre la molécule et les acides aminés du fXa constituant la zone réceptrice et, d'autre part, par un système de liaisons hydrogène. La lipophilie augmentée de la molécule $\mathrm{M}_{2}$ permet le développement d'une forme orale dotée d'une biodisponibilité suffisante.

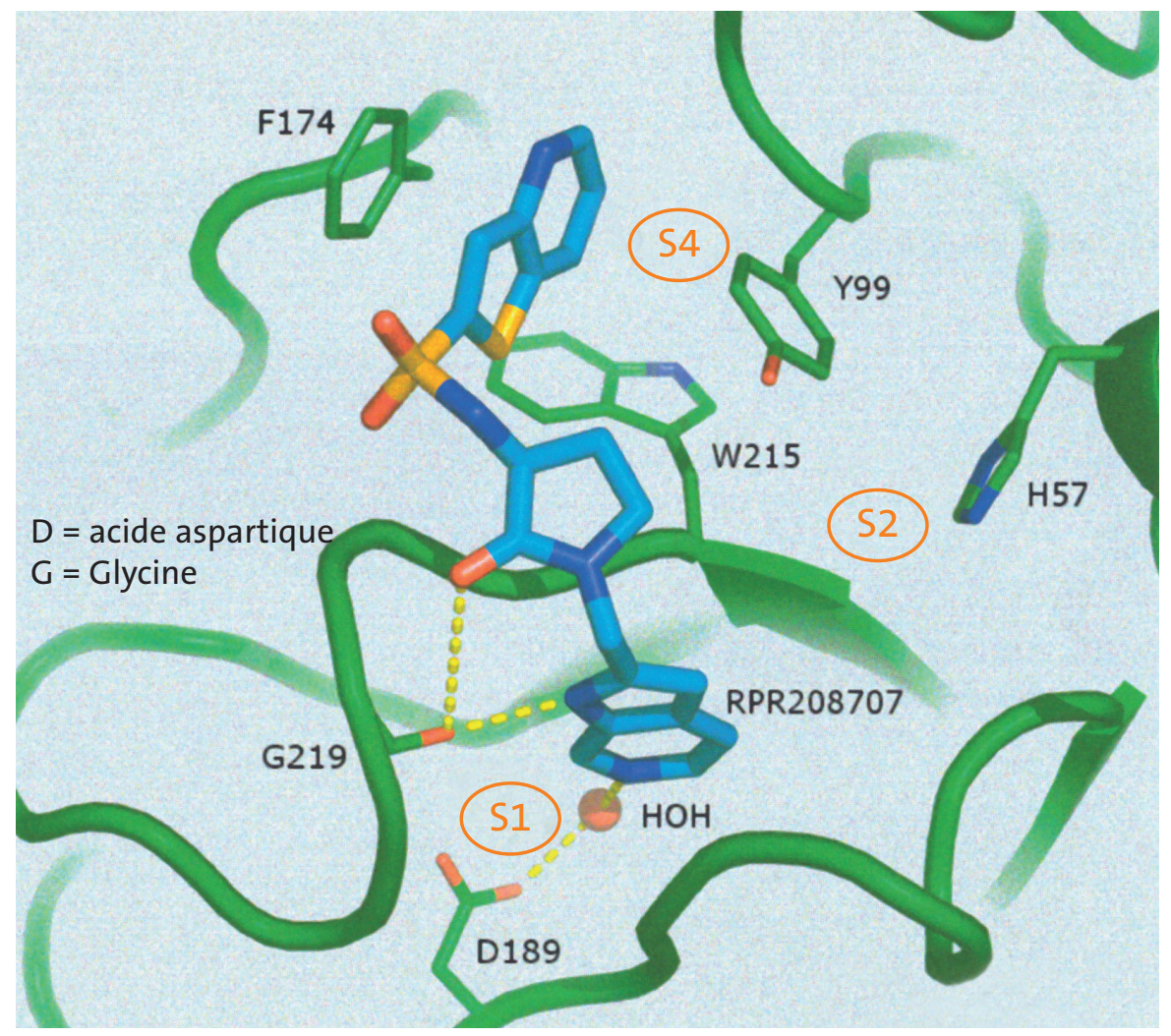

Figure 3 : Structure du complexe obtenu entre fXa et une molécule $M_{2}$ en développement. La zone réceptrice est, pour des raisons de clarté topologique, divisée en sous-zones, appelées également poches, dont trois (S1, S2 et S4) sont montrées ici. On voit en particulier que dans la poche S1, l'interaction fXa-ligand est stabilisée par un système de liaisons hydrogène (tirets jaunes), soit directes entre certaines fonctionnalités de la molécule et la glycine G219, soit par l'intermédiaire d'une molécule d'eau en interaction elle-même avec l'acide aspartique D189. Certains autres aminoacides constitutifs du fXa sont également indiqués : $F=$ phenylalanine, $H=$ histidine, $Y=$ tyrosine, $W=$ tryptophane 


\section{De l'usage de la convolution dans la mise en place d'un contrôle de qualité d'un comprimé à libération prolongée}

Rappelons le mécanisme d'absorption, par l'organisme, d'un principe actif moléculaire (M) administré par voie orale sous forme d'un comprimé ou d'une gélule. La molécule $\mathrm{M}$ se présente en général sous forme d'une poudre solide cristallisée mélangée avec d'autres produits solides inactifs, les excipients (voir introduction, p. 5). Si nous prenons le cas d'un comprimé, celui-ci, une fois absorbé par voie orale, va entrer dans le système gastro-intestinal où il va se désagréger. Progressivement, $M$ va passer de l'état solide à l'état dissous dans le milieu aqueux dont le système gastro-intestinal est constitué. Ce n'est qu'une fois à l'état de molécule dissoute que $M$ va pouvoir traverser la paroi intestinale pour se retrouver dans la circulation du sang et être amenée finalement à son site d'action. Cependant, la molécule $M$ ne va pas rester éternellement dans le sang; elle va sortir de la circulation sanguine au fur et à mesure, entre autres par excrétion urinaire.

La concentration plasmatique $\mathrm{C}(\mathrm{t}) \mathrm{de}$ $\mathrm{M}$ en fonction du temps, mesurée à partir de prélèvements sanguins consécutifs à la prise d'un comprimé, a l'allure d'une courbe en cloche dissymétrique (fig. 4). Celle-ci, appelée "pharmacocinétique " de $M$ dans la formulation sélectionnée, est le résultat de deux processus opposés : la libération in vivo de $M$ dans le sang, suivant une fonction croissante du temps $\mathrm{R}(\mathrm{t})$, et l'élimination représentée par une fonction décroissante $\mathrm{E}(\mathrm{t})$.

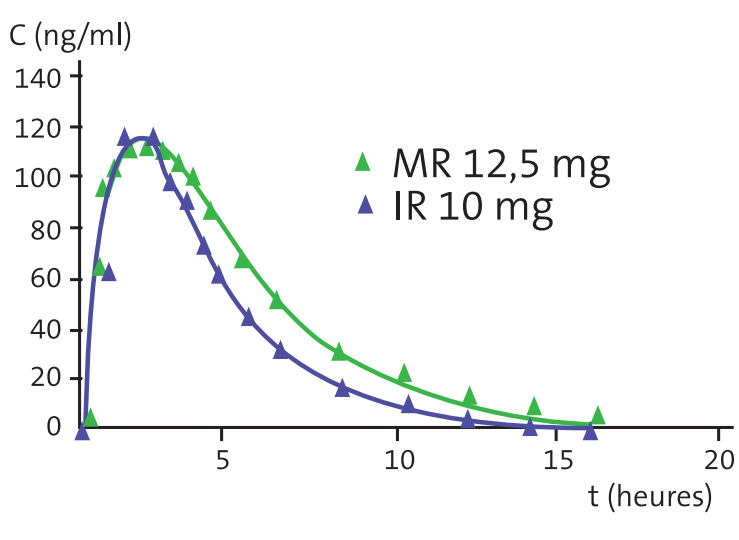

Figure 4 : Comparaison des profils pharmacocinétiques $C(t)$ obtenus pour la molécule $M$ à partir du comprimé commercialisé (triangles bleus) et $d u$ nouveau comprimé $M R$ présentant une durée d'action somnifère augmentée (triangles verts). L'idée qui a présidé à la conception de la nouvelle formulation MR a été d'associer une couche de composition identique à celle du comprimé normal et une couche préparée à partir d'un polymère gonflant en milieu aqueux gastro-intestinal, afin d'avoir un effet de libération étendu dans le temps sous l'effet d'un processus de diffusion.

La fonction d'élimination $\mathrm{E}(\mathrm{t})$ est une caractéristique comportementale intrinsèque de la molécule dans la circulation du sang et est indépendante de la forme pharmaceutique utilisée (gélules, comprimés...) et de la voie d'administration (orale, injectable, locale). Elle est obtenue une fois pour toutes à partir de mesures de la concentration sérique en $M$, après injection intraveineuse à un groupe de volontaires. Comme cette façon de faire admet une mise à disposition quasi instantanée de $\mathrm{M}$ à l'ensemble du volume sanguin, la fonction d'élimination est notée $E_{\delta}(t)$, l'indice $\delta$ rappelant le caractère "fonction impulsion " de la méthode d'administration. On peut alors montrer simplement [3, 4] que : $\mathrm{C}(\mathrm{t})=\mathrm{r}(\mathrm{t}) * \mathrm{E}_{\delta}(\mathrm{t})$, où $\mathrm{r}(\mathrm{t})=\mathrm{dR}(\mathrm{t}) / \mathrm{dt}$ est la vitesse de mise à disposition de $\mathrm{M}$ dans la circulation sanguine, et $*$ représente le symbole du produit de convolution de deux fonctions.

L'exemple que nous souhaitons traiter est une molécule $\mathrm{M}$ active contre l'insomnie et prescrite sous forme de comprimés contenant 12,5 mg de principe actif. Le but assigné par les cliniciens était que cette formulation ${ }^{\text {*: }}$ puisse présenter une
Figure 5 : Comparaison des courbes de dissolution I( $t$ ) in vitro, en vert, et de mise à disposition de la circulation sanguine $R(t)$ in vivo, en rouge. Pour permettre une comparaison directe, les résultats de la dissolution et de la mise à disposition sont exprimés en pour cent de la dose totale (12,5 mg de M), supposée passée en solution pour le test in vitro et mise entièrement à disposition dans le sang dans le cas de la courbe de pharmacocinétique. Une correction des axes de temps $(0,25$ heure) entre les deux tests est nécessaire pour tenir compte du fait que si le processus de dissolution de M s'opère immédiatement in vitro, la libération de $M$ dans le sang comporte en plus une étape limitante de diffusion de $M$ à travers la paroi intestinale.

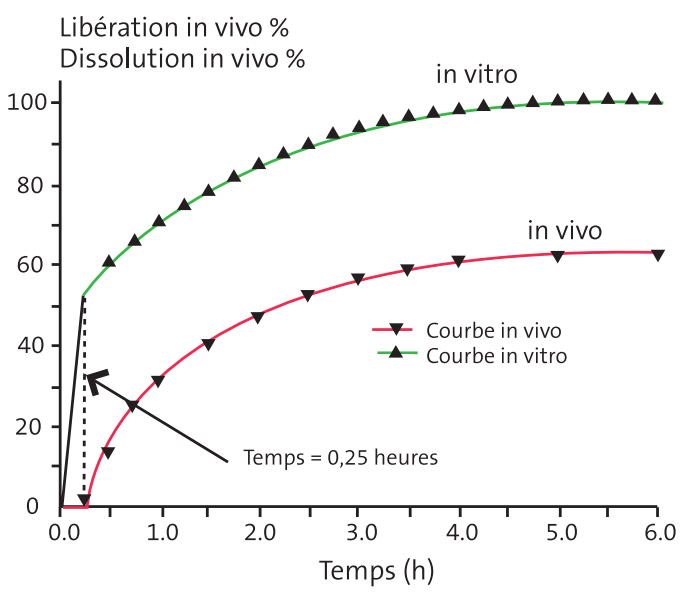

mise à disposition (ou une libération) de $M$ in vivo, telle que l'effet somnifere puisse durer au moins 5 heures. Après une mise au point très détaillée de la formulation pharmaceutique, un prototype (appelé MR pour "modified release") a été sélectionné parmi plusieurs formulations possibles, après les avoir testées chez des patients. La pharmacocinétique $\mathrm{C}(\mathrm{t})$ de $\mathrm{MR}$, représentée sur la figure 4, caractérise précisément la performance de la formulation retenue par rapport à l'objectif de durée d'action recherché.

Il va falloir maintenant produire industriellement de tels comprimés en lots atteignant le million d'unités, tout en étant sûrs qu'ils présentent le profil in vivo $\mathrm{C}(\mathrm{t})$ de la figure 4 , avec une variabilité acceptable. Comme il n'est évidemment pas question de convoquer à la fin de chaque fabrication une population de patients pour effectuer une pharmacocinétique, il va donc être nécessaire de trouver un test de remplacement in vitro, réalisable dans un laboratoire de contrôle de qualité et qui permette de libérer le lot en vue de la commercialisation. Ce test, dit de dissolution in vitro, consiste à introduire le comprimé dans un récipient contenant un milieu aqueux thermostaté à $+37^{\circ} \mathrm{C}$ et en agitation, puis à mesurer la variation temporelle, I(t), de la concentration de $\mathrm{M}$ dans la solution. La figure 5 montre la variation $\mathrm{I}(\mathrm{t}) / \mathrm{C}_{\mathrm{M}}$ de la concentration normalisée par sa valeur maximum, obtenue en supposant les 12,5 mg passés totalement en solution.

Il faut maintenant essayer de voir si I $(\mathrm{t})$ peut être corrélée avec la fonction de libération in vivo $\mathrm{R}(\mathrm{t})$ de $\mathrm{M}$ dans le sang. Une fois les fonctions $C(t)$ et $E(t)$ déterminées expérimentalement, on en déduit par déconvolution la fonction $\mathrm{r}(\mathrm{t})=\mathrm{C}(\mathrm{t}) * \mathrm{E}_{\delta}{ }^{-1}(\mathrm{t})$ puis, par intégration de $r(t)$, la fonction $R(t)$. La figure 5 compare les variations in vivo $\mathrm{R}(\mathrm{t})$ et in vitro $\mathrm{I}(\mathrm{t})$ après normalisation par $\mathrm{C}_{\mathrm{M}}$. 

$\tau$ en fonction de $1 / T$ pour le principe actif amorphe (vert), le polymère (bleu) et la préparation "hot melt" (noir). $T_{\text {melt }}$ (ici $150^{\circ} \mathrm{C}$ ) repère la température de fusion du principe actif sous sa forme cristalline.

\section{Références}

1. S. Maignan et al., "Molecular structures of factors Xa complexed with ketopiperazine inhibitors : preference for neutral group in the S Pocket", J. Med. Chem. 46 (2003) 685-690.

2. S. Maignan et al., "Crystal structures of human factor Xa complexed with potent inhibitors", J. Med. Chem. 43 (2000) 3226-3232.

3• F. N. Madden et al., "A comparison of six deconvolution techniques", J. Pharm.

Biopharm. 24 (1996) 283-299.

4- M. Pirsio et al., "A semi-parametric deconvolution model to establish in vivo-in vitro correlation applied to OROS oxybutyn", J. Pharm. Sci. 90 (2001) 702-712.

5-J. Alié et al., "Dielectric study of the molecular mobility and the isothermal crystallization kinetics of an amorphous pharmaceutical drug substance", J. Pharm. Sci. 93 (2004) 218-233.

6. J. Menegotto, M. Bauer, J. Alié, C. Mayoux, TSC and DDS, solid state characterization of pharmaceuticals, chapitre 7, édité par A. \& M. Zakrzewski (2006), assa ${ }^{\circledR}$ Inc., Danbury, Connecticut, publié par Pergamon.

7. B.C. Hancock et G. Zografi, "Characteristics and significance of the amorphous state in pharmaceutical systems", J. Pharm. Sci. 86 (1997) 1-12.

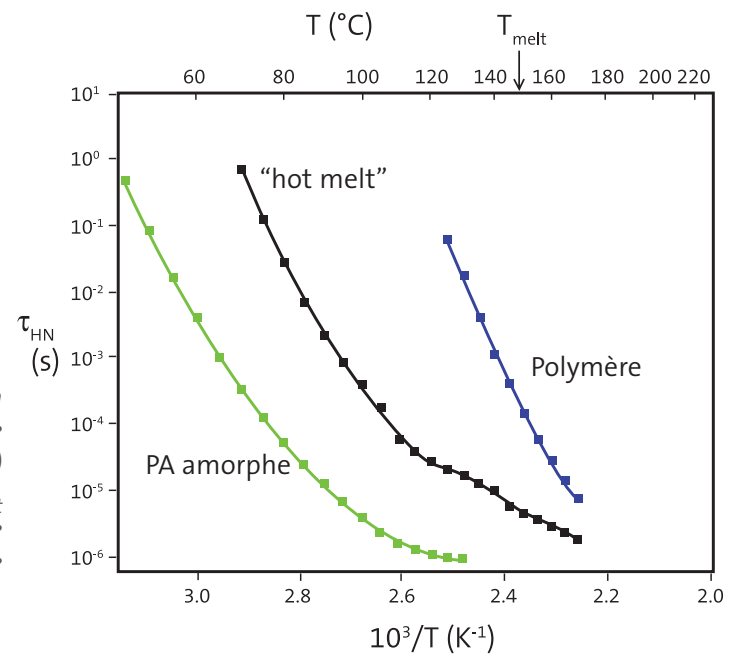

On cherche alors une fonction de corrélation in vivo-in vitro reliant de façon biunivoque $\mathrm{I}(\mathrm{t})$ et $\mathrm{R}(\mathrm{t})$ : une fonction sigmoïdale, dite de Hill, permet d'obtenir une très bonne corrélation.

Nous avons donc obtenu l'outil de contrôle de qualité que nous cherchions. L'opération se termine par la mise en place de normes d'acceptation du profil de libération in vitro. Des profils haut $\mathrm{I}^{+}(\mathrm{t})$ et bas $\mathrm{I}^{-}(\mathrm{t})$ sont préconisés comme variation maximale autorisée pour s'assurer que les profils in vivo $\mathrm{C}^{+}(\mathrm{t})$ et $\mathrm{C}^{-}(\mathrm{t})$ sont dans la marge de variation autorisée par les autorités de santé, en termes de temps correspondant au maximum et d'aire sous la courbe.

Cet exemple montre comment, grâce à une modélisation bien conduite, on peut mettre en place un contrôle de qualité efficace d'un médicament fabriqué à l'échelle industrielle.

\section{La spectroscopie diélectrique comme aide à la conception de formes pharmaceutiques}

Le dernier exemple traite la caractérisation de l'état amorphe que peut adopter un principe actif obtenu initialement sous forme cristalline. Mais pourquoi vouloir faire passer un produit parfaitement cristallisé dans un état amorphe?
Tout simplement parce que la cinétique de mise en solution in vivo d'une molécule dans le milieu aqueux du tractus gastro-intestinal est considérablement plus rapide si elle est dans un état solide amorphe (plutôt que cristallin), avec très souvent une conséquence spectaculaire sur la biodisponibilité (i.e. la fonction $r(t)$ de l'exemple précédent). Or, il se trouve que les molécules issues de la recherche fondamentale sont intrinsèquement de moins en moins solubles dans l'eau avec, par conséquent, le risque d'avoir une faible biodisponibilité. On peut, dans le même état d'esprit, préparer une forme pharmaceutique en dispersant la molécule active dans un polymère amorphe hydrophile, formant ainsi un système monophasé amorphe. L'absence d'étape de destruction du réseau cristallin va favoriser l'accélération de la mise en solution, voire la biodisponibilité.

L'étude de ces systèmes très élaborés nécessite l'utilisation de méthodes physiques comme la spectroscopie diélectrique dynamique $[5,6]$, qui permet de mettre en évidence les différentes mobilités d'une molécule, suivant qu'elle se trouve dans un état amorphe, semi-cristallin ou cristallin. En bref, cette technique consiste à placer entre deux plaques d'un condensateur, à une température T, l'échantillon à étudier et à soumettre l'ensemble à une différence de potentiel sinusoïdale, dont on peut faire varier la fréquence f entre $10^{-1}$ et $10^{6} \mathrm{~Hz}$. On enregistre la variation de l'angle de perte $\operatorname{tg} \delta=\varepsilon " / \varepsilon$ ' en fonction de $\mathrm{f}$ et de T. Les entités $\varepsilon$ " et $\varepsilon$ ' sont les deux termes qui interviennent dans la permittivité complexe $\varepsilon$ du matériau définie comme $\varepsilon(\mathrm{f})=\varepsilon^{\prime}(\mathrm{f})+i \varepsilon^{\prime \prime}(\mathrm{f})$. Le terme $\varepsilon^{\prime}$ est relié à l'état de polarisation des dipôles de l'échantillon induit par le champ électrique alternatif ; le terme $\varepsilon$ " est relié à l'énergie dissipée durant les mouvements des dipôles sous l'action du champ électrique.

Les paramètres $\varepsilon^{\prime}, \varepsilon$ " et donc tg $\delta$ sont l'expression macroscopique de la relaxation des différentes familles de dipôles constituant le matériau analysé. Ces processus de relaxation

\section{La recherche et développement à Sanofi-aventis}

- 17600 personnes réparties sur 25 centres de R\&D et 3 continents. En France, les principaux centres se situent dans la région parisienne, ainsi qu'à Montpellier, Strasbourg et Toulouse.

- Principaux axes de recherche : le cardio-vasculaire, la thrombose, le cancer, les maladies métaboliques, la médecine interne, les vaccins.

- Principaux équipements de caractérisation physique des préparations pharmaceutiques : RMN du solide, microscopie électronique couplée à l'analyse en énergie des rayons X émis, microscopie Raman confocale, spectroscopies diélectriques..

- Une dizaine de partenariats avec des entreprises de biotechnologie ou des organismes de recherche publics et universitaires dans des domaines touchant aux anticorps monoclonaux et vaccins thérapeutiques, en particulier dans le domaine du cancer.

- La R\&D accueille des stagiaires (par exemple, une centaine chaque année à Montpellier) de différentes origines scolaires et universitaires dans le cadre de travaux de fin d'études ou de thèses, dans les divers domaines concernés par les axes de recherches. 
sont associés à des temps caractéristiques $\tau$ qui traduisent l'échange d'énergie des entités relaxantes avec leur environnement, et qui, comme dans tous les processus activés, dépendent de la température. On peut concevoir que, selon la nature cristalline ou amorphe de l'échantillon, ces temps de relaxation seront différents, ainsi que leur variation en fonction de la température. Il est possible, à partir de l'analyse mathématique des surfaces $\operatorname{tg} \delta(f, T)$, d'extraire à l'aide de divers modèles théoriques $[5,6]$ la variation $\tau(T)$ qui nous intéresse pour caractériser un système donné. Certains mouvements sont décrits par une variation linéaire de $\ln \tau$ en fonction de $1 / \mathrm{T}$ (comportements de type Arrhenius). D'autres modes de relaxation, en particulier ceux impliqués dans une transition vitreuse, correspondent à une variation de $\ln \tau(\mathrm{T})$ non linéaire avec $1 / \mathrm{T}$.

Nous avons appliqué cette approche à une formulation obtenue après dissolution d'un principe actif, particulièrement insoluble dans l'eau, dans un polymère hydrosoluble en chauffant le mélange au-dessus de la température de transition vitreuse $\mathrm{T}_{\mathrm{g}}$ du polymère. L'ensemble est ensuite ramené à̀ la température ambiante (formulation dite "hot melt"). Le polymère et le principe actif sont dans un rapport massique 50/50. La carte de relaxation $\operatorname{tg} \delta(f, T)$ de cette formulation présente plusieurs modes de relaxation. Dans le cas présent, nous nous sommes intéressés au mode associé à la transition vitreuse. La figure 6 récapitule les variations de $\log \tau$ en fonction de $1 / \mathrm{T}$ pour le polymère hydrophile amorphe seul, le principe actif seul et la formulation "hot melt" $(50 / 50)$. On peut constater que la courbe $\tau(\mathrm{T})$ de la formulation "hot melt" est décroissante monotone comme celles des composants seuls, et est située entre ces deux dernières. Un processus unique de relaxation est ainsi détecté pour le mélange "hot melt", ce qui est la signature univoque du caractère monophasique de cette formulation. Le principe actif se trouve donc à l'état de dispersion moléculaire dans le polymère, ce qui était le but recherché. Il a bien sûr été vérifié par des essais in vitro, que la dissolution du principe actif dans la formulation "hot melt" était considérablement accélérée par rapport à celle du principe actif sous forme cristalline. Ce type de développement fait l'objet d'un intérêt soutenu dans la recherche pharmaceutique [7].

\section{Conclusion}

Nous espérons que ces quelques exemples illustrent combien la R\&D pharmaceutique a aujourd'hui besoin de chercheurs formés à la physique et aux mathématiques. Bien d'autres exemples auraient pu renforcer cette constatation. C'était le message que nous avons souhaité adresser aux étudiants-chercheurs dans le cadre des $10^{\text {es }}$ Journées de la matière condensée.

\section{Remerciements}

Je tiens à remercier Sandrine Bascou, Sébastien Maignan, Estelle Weinling, Véronique Serre, Jérôme Menegotto et Jean Alié pour leur aide précieuse dans la préparation de cet article.

\section{Glossaire}

Biodisponibilité

On appelle biodisponibilité d'une

molécule pharmaceutique $M$, sa

mise à disposition dans le sang à

partir d'une prise orale.

\section{Constante d'affinité}

La constante d'affinité $K_{D}$ d'un complexe protéine (P)-molécule ligand $(M)$ en solution aqueuse à la température T est la constante d'équilibre de l'équation de complexation $P-M \leftrightarrow P+M$ : $K_{D}(T)=[P][M] /[P-M]$.

Une valeur de $K_{D}$ inférieure à 50 nmol. I $^{-1}$ correspond à une très grande affinité du ligand pour la protéine réceptrice.

\section{Formulation}

La formulation pharmaceutique correspond à la description détaillée, qualitative et quantitative, des différents composants d'un médicament (principe(s) actif(s)-excipients), ainsi que de son procédé de fabrication. Un médicament peut être administré par différentes voies et sous différentes formes physiques:

- voie orale : formes solides (comprimés, gélules), suspensions, solutions buvables ;

- voie parentérale : solutions injectables intraveineuses ou intramusculaires ;

- voies locales : dermique (crèmes, lotions, patchs...), nasale ou pulmonaire (aérosols, gouttes...) ; - voie rectale (suppositoires). 